\title{
EFFECT OF A SUBWAVELENGTH LAYER ON ALL OPTICAL-DIODE ACTION IN 1D PHOTONIC CRYSTAL
}

\author{
KAZEM JAMSHIDI-GHALEH \\ Department of Physics, Azarbaijan University of Tarbiat Moallem, Tabriz, Iran \\ k-jamshidi@azaruniv.edu \\ ZEINAB SAFARI \\ Department of Optics-Laser, University of Bonab, Azarbaijan-e Shargi, Bonab, Iran \\ z.safari@yahoo.com
}

\begin{abstract}
In this paper, the effect of a subwavelength layer thickness with positive-refractive index material on all-optical diode action in a one-dimensional photonic crystal structure is investigated. An asymmetric multilayer stack composed of Kerr-type nonlinear and linear dielectric media and a subwavelength layer is considered. Behaviour of the linear transmission defect mode and nonlinear optical bistabilities for normal incidence of wave to $1 \mathrm{D}$ photonic crystal structure from left and right sides are studied. It is demonstrated that, with increasing of the subwavelength layer thickness, the linear defect mode shifts to the lower frequencies. Also, the left to right one-way transmission property of the structure is changed for right to left transmission at some subwavelength layer thicknesses. The changes of lower and higher bistability intensities thresholds and difference between left to right and right to left nonlinear transmissions versus subwavelength layer thickness are graphically illustrated. The photonic crystal all-optical diode is one of the important integrated devices, which has potential applications in the field of optical computing, optical interconnection systems and integrated photonic circuits.
\end{abstract}

Keywords: All-optical diode; optical bistability; photonic crystal, defect mode.

\section{Introduction}

In recent years, the artificial periodic structures, so called Photonic Crystals (PCs) firstly introduced by Yablonovich [1] and John [2], are designing to control and manipulate the electromagnetic waves propagation. The stop band known also as photonic or forbidden band gape is a remarkable feature of the PC structure which is similar to the electronic band gap in a semiconductor. The electromagnetic field of the frequency within the band gap is evanescent. This conventional photonic band gap originates from the interference of Bragg scattering due to the periodicity of the structure and highly sensitive to the periodic constant, incident angle and state of polarization. It is also affected by disorder, randomness and thickness tolerances. In last two-decades, due to special electromagnetic performance and potential applications of the PCs, more attentions are attracted to design the devices capable to controlling of the photons [3-8] by the PCs. Incorporating of the 
nonlinearity with PC structures one has the capability to control wave propagation dynamically. Optical bistability (OB) is one of the ways of manipulating the transportation of photons and can be used in designing of an all-optical switching $[9,10]$. OB can be induced by dynamic shifting of the band edge or one of the resonant modes $[11,12]$, which appears in the photonic band gap of a defective periodic or disordered PC structure. Inouyea and Kanemitsu experimentally observed mode shifting and opened the new fields in application of PCs in the nonlinear optical devices [13]. The bistability of one-dimensional (1D) PCs containing negative-index materials has also attracted much attention. The positive-negative-index periodic structures, containing a single nonlinear defect, have demonstrated tunable bistable switching and transmission [14]. It has been reported that introducing of a positive or negative-refractive index subwavelength thin layer (SWL) in a 1D PC structure significantly affects the bistability thresholds $[15,16]$. The OB performance of a spatially asymmetric PC structure together with anisotropy of the field intensity distribution in layers puts the structure to be a device that is called alloptical diode (AOD). AOD permits to pass an electromagnetic wave from one side of the structure but not from the other side and is an electronic analogue of the electric diode that passes electricity from one side only. AOD has promising application in some areas such as optical isolation and all optical signal processing. In the last years, most attentions are directed to design new AOD structures or to optimize its efficiency.

In this paper, we have investigated the effect of a SWL on AOD action in an asymmetric 1D PC. We have shown that the one-way transmission property (diode action) from the structure can be manipulated by varying of the SWL thickness. In section 2 the 1D PC under study and the optical constant of the materials are given. A short and brief review of the necessary mathematical formulas is presented. The results of numerical calculations based graphical illustrations are given in section 3 . The results are summarized in section 4 .

\section{Model and Theory}

The 1D PC structure under consideration is indicated by $(\mathrm{BA})^{5}(\mathrm{AB})^{5} \mathrm{C}(\mathrm{AABB})^{5}$. Here letters $A$ and $B$ stands for nonlinear material with $\mathrm{n}_{\mathrm{A}}=1.55$ and $\chi_{\mathrm{A}}=2.5 \times 10^{-5} \mathrm{~cm}^{2} / \mathrm{MW}$ (polydiacetylene 9-BCMU), and linear material with $\mathrm{n}_{\mathrm{B}}=2.3\left(\mathrm{TiO}_{2}\right)$, respectively [17]. Here, we have used 5 for the number of periodicity in the stack. The letter $\mathrm{C}$ denotes the SWL with optical thickness of a fraction of wavelength and can be a negative or positive index material. In our study, the positive index material with linear refractive index of $\mathrm{n}_{\mathrm{c}}=1.55$ is used (Crown glass series with damage threshold of the order of $10^{14} \mathrm{~W} / \mathrm{cm}^{2}$ ). The optical thickness of layers $A$ and $B$ are the same, that is: $n_{A} d_{A}=n_{B} d_{B}$. We have defined a characteristic wavelength of $\lambda_{0}=4 n_{A} d_{A}=700 \mathrm{~nm}$. The well-known simple and powerful transfer matrix method $[18,19]$ is employed to analysis the normally propagation of the electromagnetic wave through the PC structure. According of this method, the normally incident electric $E$ and magnetic $H$ fields at the opposite sides of each single layer can be related in the following way: 


$$
\begin{aligned}
& \left(\begin{array}{c}
E \\
i H
\end{array}\right)_{j}=M_{j}\left(\begin{array}{c}
E \\
i H
\end{array}\right)_{j+1} \\
& M_{j}=\left[\begin{array}{cc}
\cos \left(\frac{n_{j} d_{j} \omega}{c}\right) & n_{j}^{-1} \sin \left(\frac{n_{j} d_{j} \omega}{c}\right) \\
-n_{j} \sin \left(\frac{n_{j} d_{j} \omega}{c}\right) & \cos \left(\frac{n_{j} d_{j} \omega}{c}\right)
\end{array}\right]
\end{aligned}
$$

where, $d_{j}$ and $n_{j}$ are the thickness and refractive index of the layers, respectively, $\omega$ is the frequency and $\mathrm{c}$ is the speed of light. We have assumed that all materials are dispersionless and the refractive indices of the layers are frequency independent. The total transfer matrix of the whole structure can be obtained by multiplying together all transfer matrices of subsequent layers

$$
M=M_{1} M_{2} \ldots M_{N-1} M_{N}
$$

and the transmission spectrum in terms of the total matrix elements is given by

$$
T(\omega)=\frac{4}{\left|M_{11}+M_{22}-i\left(M_{21}-M_{12}\right)\right|^{2}}
$$

\section{Results and Discussions}

Fig. 1(a) shows the behaviour of the linear transmission spectrum around of the defect mode at four different thickness of SWL, depicted on the figure, where the frequency is scaled in units of $\omega_{0}=2 c \pi / \lambda_{0}$. It is seen clearly, with increasing of the SWL thickness the defect mode shits to the lower frequencies (inset of Fig. 1(a)).
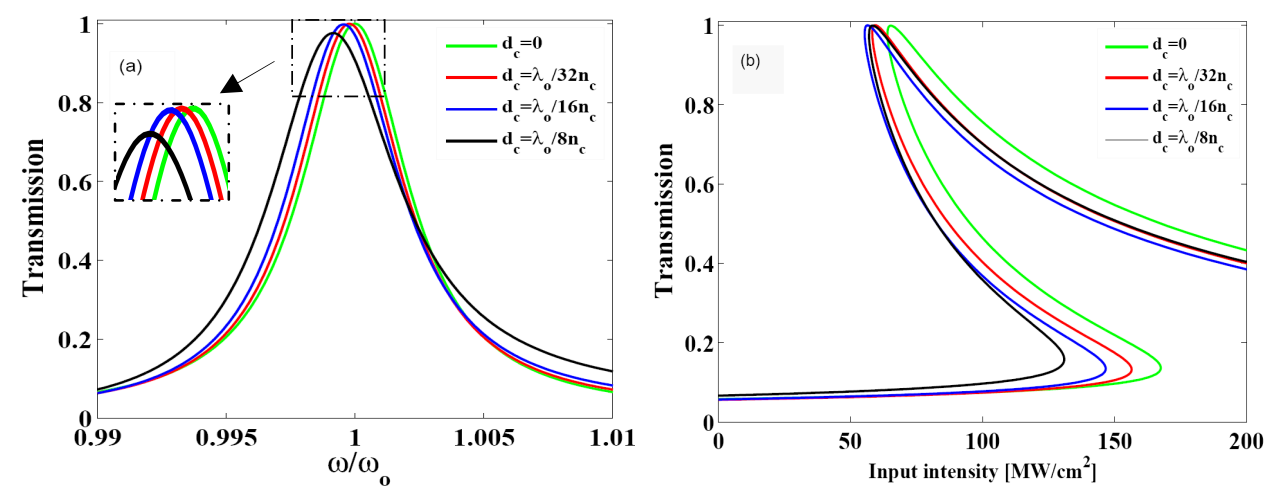

Fig. 1. Behaviour of the defect mode linear transmission (a) and nonlinear OB (b) of the $(\mathrm{BA})^{5}(\mathrm{AB})^{5} \mathrm{C}(\mathrm{AABB})^{5}$ structure for normal incident corresponding to the SWL optical thicknesses of $0, \lambda_{0} / 32 \mathrm{n}, \lambda_{0} / 16 \mathrm{n}$ and $\lambda_{0} / 8 \mathrm{n}$. The inset shows the close view of the transmission near the peak value.

For smaller thickness the defect mode peak heights remain constant (transmission is perfect), but further increasing of the thickness, leads to the reduction of the transmission. Although the amount of reduction is small but it is very important on the action of an OAD. The perfect transmission of the defect mode is a necessary condition for high 
contrast performance of the AOD. Therefore, the thicknesses thicker than the $\lambda_{0} / 16 n_{c}$ can not be considered as a SWL. Also, the frequency shift is small but can significantly affect the threshold intensity of the OB. Fig. 1(b) shows the behaviour of the nonlinear transmission versus input intensity for four different thicknesses of the SWL.
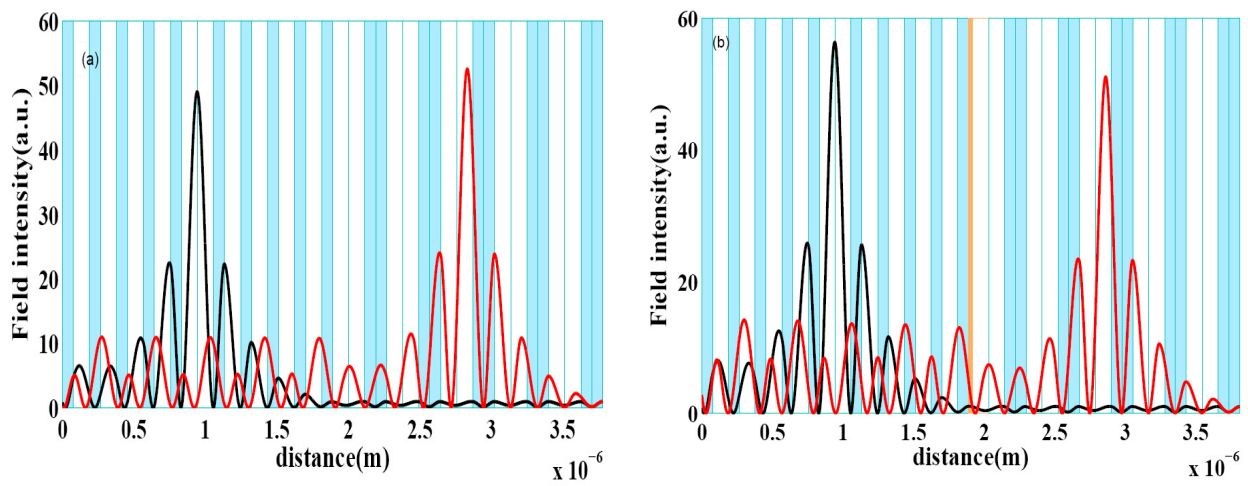

Fig. 2. Field distribution inside the layers of the $(\mathrm{BA})^{5}(\mathrm{AB})^{5} \mathrm{C}(\mathrm{AABB})^{5}$ for $d_{c}=0$ (a) and $d_{c}=\lambda_{0} / 16 n_{c}$ (b).

One of the necessary conditions for a PC structure that can possess AOD action is that the field intensity distribution inside the layers should be anisotropic from left and right side incidence of the beam. Fig. 2 shows the electric field distribution inside layers of the structure under consideration, for left side (black) and right side (red) beam incidence, without SWL (Fig.2(a)) and for thickness of $d_{c}=\lambda_{0} / 16 n_{c}$ (Fig.2(b)). In the absence of the SWL, the field distribution have a mirror symmetry in respect to center of the structure but by inserting the SWL the localization of the field from left side beam incidence is higher than the right side one.


Fig. 3. OBs from left and right sides beam incidences for $d c=0$ (a) and $d_{c}=\lambda_{0} / 16 n_{c}$ (b).

The $\mathrm{OB}$ of the $(\mathrm{BA})^{5}(\mathrm{AB})^{5} \mathrm{C}(\mathrm{AABB})^{5}$ structure from left side (black) and right side (red) beam incidence for two cases of $d_{c}=0$ (Fig. 3(a)) and $d_{c}=\lambda_{0} / 16 n_{c}$ (Fig. 3(b)) are plotted in 
Fig.3. In the absence of SWL the threshold intensities (switch up and switch down intensities) for left side incidence are higher than for the right side incidence but for $\mathrm{d}_{\mathrm{c}}=\lambda_{0} / 16 \mathrm{n}_{\mathrm{c}}$, the right side beam incidence thresholds are higher than the left side ones. Fig.4 illustrates the higher (Fig. 4(a)) and lower (Fig. 4(b)) OB intensity thresholds for L$\mathrm{R}$ (blue) and R-L (red) beam incidences. For both cases, in the absence as well as for higher thickness of the SWL, the L-R threshold is higher than the R-L one. But for thickness range from $d_{c}=\lambda_{0} / 32 n_{c}-d_{c}=\lambda_{0} / 16 n_{c}(14 n m-34 n m)$ they show the inverse behaviour.


Fig. 4 The higher (a) and lower (b) OB intensity thresholds with increasing of the SWL thickness

Fig. 5, shows the behaviour of L-R and R-L bistabilities threshold difference with increasing of the SWL thickness. As it is shown, at the thicknesses range of $d_{c}=\lambda_{0} / 32 n_{c}-$ $\lambda_{0} / 16 \mathrm{n}_{\mathrm{c}}$, it accepts the negative values, indicating that the $\mathrm{L}-\mathrm{R}$ one way transmission is changed to R-L one. This property can be useful on the controlling of the wave propagation by next coming all-optical integrated circuits.



Fig. 5. Behaviour of the $[(L-R)-(R-L)]$ intensity thresholds versus the SWL thickness 


\section{Conclusion}

We have demonstrated that by inserting of a SWL in the 1D PC structure, the all-optical diode action can be controlled. We have used the arrangement of $(\mathrm{BA})^{5}(\mathrm{AB})^{5} \mathrm{C}(\mathrm{AABB})^{5}$, where layers $A$ and $B$ are the nonlinear and linear materials, respectively, and $C$ is the SWL with positive-index linear material. In numerical calculations, we have used to $\mathrm{n}_{\mathrm{A}}=1.55, \chi_{\mathrm{A}}=2.5 \times 10^{-5} \mathrm{~cm}^{2} / \mathrm{MW}, \mathrm{n}_{\mathrm{B}}=2.3, \mathrm{n}_{\mathrm{c}}=1.55$ and $\lambda_{0}=4 \mathrm{n}_{\mathrm{A}} \mathrm{d}_{\mathrm{A}}=4 \mathrm{n}_{\mathrm{B}} \mathrm{d}_{\mathrm{B}}=700 \mathrm{~nm}$. The effect of SWL $C$ thickness on linear transmission defect mode and nonlinear optical bistabilities for left to right and right to left beam incidences are investigated. The redshift of the linear defect mode frequency is observed with increasing of the thickness. The L-R one-way transmission is changed to the R-L transmission at the range of the thickness $d_{c}=\lambda_{0} / 32 n_{c}-\lambda_{0} / 16 n_{c}(14 n m-34 n m)$. The behaviours of lower and higher bistability threshold intensities and difference between L-R and R-L nonlinear transmissions versus of the SWL thickness are graphically illustrated. Photonic crystal AOD is one of the important devices, which has potential applications in the field of optical computing, optical interconnection systems and integrated photonic circuits.

\section{References}

1. E. Yablonovich, Phys. Rev. Lett. 58 (1987) 2059.

2. S. John, Phys. Rev. Lett. 58 (1987) 2486.

3. J.D. Joannopoulos, S. Johnson, R. Meade, J. Winn, Photonic Crystals, (University Press, Princeton, 2008)

4. T.F. Krauss, R.M. De La Rue, Progress in Quantum Electronics, 23 (1999) 51

5. S. Arishmar Cerqueira Jr, Rep. Prog. Phys. 73 (2010) 024401

6. L. Joshing, Optics Communication, 283, 13, 1 (2010) 2647

7. R. Asadi, M. Malek-Mohammad and S. Khorsandi, Optics Communication, 284, 8, 15 (2011) 2230

8. M.Y. Mahmoud, G. Bassou, A. Talbi, Z.M. Chekrou, Optics Communication, (in press) doi: 10.1016/j.optcom.2011.09.068

9. E. Wolf, Progress in Optics, (North Holland Physics Publishing, Amesterdam, 1984)

10. J. He, M. Cada, IEEE J. Quantum Electron. QE-27, 1182 (1991)

11. P. Tran, Opt. Lett. 21 (1996) 1138.

12. R. Wang, J. Dong, D.Y. Xing, Phys. Rev. E 55 (1997) 6301.

13. H. Inouyen, Y. Kanemitsu, Appl. Phys. Lett. 82 (2003) 1155.

14. M.W. Feisem I.V. Shadrivov, Y.S. Kivshar, App. Phys. Lett. 85 (2004) 1451.

15. P. Hou, Y. Chen, J. Shi, Q. Kong, L. Ge and Q. Wang, Appl. Phys. A 91 (2008)41.

16. P. Hou, Y. Chen, J. Shi, M. Shen, X. Chen and Q. Wang, Europhys. Lett. 81 (2008) 6401.

17. S.V. Zhukovsky and A.G. Smirnov, Phys. Rev. A 83 (2011) 023818

18. P. Yeh, Optical Waves in Layered Media (New York: Wiley, 2005).

19. J. Li, L. Zhou, C.T. Chan and P. Sheng, Phys. Rev. Lett. 90 (2003) 083901. 